\title{
Suscetibilidade antimicrobiana de uropatógenos em pacientes ambulatoriais na Cidade de Goiânia, G0
}

\author{
Antimicrobial susceptibility of the uropathogens in out patients \\ in Goiânia City, Goiás State
}

\author{
Karine Queiroz Poletto ${ }^{1,2}$ e Cleomenes Reis ${ }^{3}$
}

\begin{abstract}
RESUMO
Em função do aumento de microrganismos multirresistentes causadores de cistite, este estudo teve como objetivo identificar os patógenos causadores de infecção do trato urinário em mulheres, além de traçar o perfil de suscetibilidade antimicrobiana. Foram analisadas 442 amostras de urina, no período compreendido entre junho de 2002 e agosto de 2003. A identificação dos germes isolados foi realizada por provas bioquímicas e enzimáticas e 0 antibiograma pelo método de difusão em disco. Ocorreram quadros laboratoriais de infecção do trato urinário em 17,6\% dos casos. Foi observada a Escherichia coli como o microrganismo prevalente causador de infecção do trato urinário (67,9\%). As bactérias Gramnegativas foram resistentes à amoxicilina em 74,6\% dos casos analisados, sendo sensíveis em maior índice a ceftazidima e gentamicina. As bactérias Gram-positivas foram resistentes em maior índice à ampicilina (72,7\%), sendo sensíveis a trimetoprim/sulfametoxazol, vancomicina e linezolida. Concluiu-se que o estudo da resistência bacteriana é necessário para indicar novas opções terapêuticas.
\end{abstract}

Palavras-chaves: Infecção urinária. Exame de urina. Bactérias Gram-positivas e Gram-negativas. Escherichia coli.

\begin{abstract}
Due to the fact that the number of multiresistant microorganisms which cause cystitis is increasing, the objective of this study was to identify the pathogens that cause urinary tract infection in women and determine antimicrobial susceptibility. We analyzed 442 urine samples from June 2002 to August 2003. Identification through biochemical and enzymatic assays, and the antibiogram by Disk diffusion method were performed. Out of these samples, 78 were positive for urinary tract infection (17.6\%). Escherichia coli were the microorganisms that most frequently caused infections (67.9\%). Gram-negative bacteria exhibited resistance to amoxicillin in $74.6 \%$ of the cases. Most were ceftazidime and gentamicin sensitive. Gram-positive bacteria were resistant in $72.7 \%$ of the samples to ampicillin, and they were trimethoprim/sulfamethoxazole, vancomycin and linezolid sensitive. It was concluded that investigation of bacterial resistance is necessary to provide information for new therapeutic options.
\end{abstract}

Key-words: Urinary infection. Urine test. Gram-positive and Gram-negative bacteria. Escherichia coli.

A infecção urinária consiste na colonização microbiana da urina com a invasão tecidual de qualquer estrutura do trato urinário ${ }^{3738}$. Os microrganismos podem chegar ao trato urinário por meio de três vias: ascendente, hematogênica e linfática ${ }^{31}$.

No Brasil, um total de $80 \%$ das consultas clínicas devem-se à infecção do trato urinário (ITU) ${ }^{26}$. De acordo com Palma \&
Dambros $^{32}$, as cistites representam um problema de saúde na mulher, afetando entre 10\% e 20\% destas durante suas vidas, sendo que $80 \%$ apresentam infecções recorrentes. Entretanto, Gupta cols ${ }^{13}$ afirmam que aproximadamente 50 a $70 \%$ das mulheres apresentam pelo menos um episódio de ITU em suas vidas, sendo que, 20 a $30 \%$ destas apresentam episódios recorrentes. No entanto, a real

\footnotetext{
1. Departamento de Microbiologia do Instituto de Patologia Tropical e Saúde Pública da Universidade Federal de Goiás, Goiânia, G0. 2. Departamento de Microbiologia, Parasitologia e Histologia da Fundação Universidade Regional de Gurupi, TO. 3. Departamento de Microbiologia do Instituto de Patologia Tropical e Saúde Pública da Universidade Federal de Goiás, Goiânia, GO.

Endereço para correspondência: Dra. Karine Queiroz Poletto. Av. Pará 1727, Centro, 77403-010 Gurupi, T0.

Tel: 5563 312-4015; 5563 9984-9512 Fax: 5563 312-3500.

e-mail: karinepoletto@uol.com.br

Recebido para publicação em 13/10/2004

Aceito em $3 / 5 / 2005$
} 
incidência de ITU é, provavelmente, subestimada, porque pelo menos metade de todas as infecções urinárias se resolve sem atenção médica ${ }^{39}$.

A ocorrência do patógeno causador de ITU varia geograficamente e 0 perfil de suscetibilidade requer monitoramento para fornecer informações para novas orientações de opções terapêuticas ${ }^{11}$. Uma das principais preocupações quanto ao uso de medicamentos está relacionada à utilização de antimicrobianos. 0 aumento da resistência bacteriana a vários agentes antimicrobianos acarreta dificuldades no controle de infecções e contribui para 0 aumento dos custos do sistema de saúde e dos próprios hospitais?.

Amaioria dos trabalhos acerca do isolamento e identificação de cepas bacterianas multirresistentes foi realizada em pacientes hospitalizados, entretanto, acredita-se que microrganismos resistentes possam ser agentes de ITU, também, na comunidade. Devido à carência de estudos neste sentido, o presente trabalho teve como objetivos a identificação dos patógenos causadores de infecção urinária em mulheres atendidas em ambulatório residentes em Goiânia, G0, traçar o perfil de suscetibilidade antimicrobiana das espécies bacterianas encontradas em mulheres com ITU e fazer a verificação da sensibilidade e da resistência dos isolados, em testes realizados pela automação (MicroScan - DaDe Behring) e difusão em disco (Kirby-Bauer).

\section{MATERIAL E MÉTODOS}

População de estudo. 0 estudo foi realizado a partir de amostras de urina recebidas no Laboratório de Análises da Saúde da Universidade Católica de Goiás ( LAS/UOC) , localizado em GoiâniaGoiás, no período compreendido entre junho de 2002 e agosto de 2003. Participaram da pesquisa 442 mulheres. Todas as pacientes consentiram em participar do trabalho. 0 projeto presente foi aprovado pela Comissão de Ética da Universidade Católica de Goiás.

Coleta da amostra. A coleta da urina foi feita segundo descrição de $\operatorname{Kunin}^{19}$. 0 processamento laboratorial foi feito sempre dentro de 60 a 90 minutos.

Identificação microbiana. Foram utilizadas amostras de urina não centrifugadas, sendo homogeneizadas e submetidas à coloração pelo Gram $^{12}$. Para 0 procedimento de contagem de colônias, foi feita a inoculação da urina, através da técnica de semeadura em estrias, em ágar CLED (ágar bromotimol-azul lactose cistina - Merck). Após 18 a 24 horas a $36,5^{\circ} \mathrm{C}$ foi estimado o número de bactérias, sendo consideradas positivas as amostras com contagem de colônias superiores a 100.000, ou seja, $\geq 10^{5} \mathrm{ufc} / \mathrm{ml}^{18}$.

Das amostras com contagem superiores a $10^{5} \mathrm{ufc} / \mathrm{ml}$, realizou-se esgotamento de alça no ágar MacConkey (Merck), se Gram-negativo; e em blood ágar base no 2 desidratado ( Difco) acrescido de sangue de carneiro 5\% e Ágar Manitol Sal (Merck), se Gram-positivo, obtendo-se assim, após incubação da amostra por 18 a 24 horas a $36,5^{\circ} \mathrm{C}$, colônias isoladas ${ }^{18}$.

As bactérias Gram-negativas foram identificadas por meio dos seguintes métodos: fermentação do ágar tríplice açúcar- ferro (TAF) ; produção de urease; fenilalanina desaminase; oxidação fermentativa de arabinose; inoculação em meio MIO (Motilidade, Indol, Ornitina) e LA (Lisina, Indol, Arginina); Vermelho de Metila; Voges-Proskauer (VP); capacidade de multiplicação em citrato de Simmon's; provas de gelatinase e DNAse ${ }^{18}$.

As bactérias Gram-positivas foram submetidas à: prova da catalase; coagulase em lâminas e em tubos; sensibilidade à novobiocina e bacitracina; fermentação de trealose e manitol; hidrólise do hipurato de sódio; bílis esculina; cloreto de sódio a 6,5\% ( NaCl 6,5\%) e PYR (L-pirrolidonil $\beta$-nattilamida) ${ }^{18}$.

Os microrganismos identificados pelas técnicas clássicas, tiveram sua identidade observada pelo aparelho automatizado MicroScan (Dade Behring, West Sacramento, Califórnia, USA) no Laboratório de Análises da Saúde - Universidade Católica de Goiás (LAS/UCG). Todos os isolados que se desenvolveram em ágar MacConkey foram identificados pelo painel Urine Combo 6 e os isolados em Blood ágar base n- 2 desidratado acrescido de sangue de carneiro $5 \%$ e ágar manitol salgado foram identificados utilizando o painel Pos Combo 21. Os painéis foram processados usando 0 sistema AutoScan $4^{922} 36$.

Prova de suscetibilidade a antimicrobianos. Antibiograma: para a realização do antibiograma pelo método de difusão em disc $0^{6}$, foi utilizado o ágar Müeller-Hinton (Merck). Na presença de estreptococos e enterococos, foi acrescido ao ágar Müeller-Hinton, sangue de carneiro a 5\%, em função da exigência para o desenvolvimento. 0 inóculo foi preparado através de solução salina ( cloreto de sódio 0,85\% ( Synth). Adensidade da suspensão foi ajustada em cerca de $10^{8}$ unidades formadoras de colônias (ufc) por mililitro, comparando sua turvação com a metade do padrão 1 da escala de MacFarland. 0 inóculo foi distribuído, através de varredura utilizando swab de Rayon (INLAB Diagnóstica), na superfície do ágar Müeller-Hinton. Em seguida, foram colocados os discos de antimicrobianos no ágar com auxílio de uma pinça estéril. Após a aposição dos discos, as placas foram invertidas e incubadas a $36,5^{\circ} \mathrm{C}$ por 18 a 24 horas. Após este período, as placas foram analisadas através da medida dos halos de inibição do microrganismo frente à droga testada, segundo a NOCLS ${ }^{27}$, utilizando-se os discos de papel fabricados pela CECON ( São Paulo, Brasil) e apenas a Linezolida fabricada pela OXOID (Basingstoke, England).

Frente às bactérias Gram-negativas, foram testados os seguintes antimicrobianos: amoxicilina $(10 \mu \mathrm{g})$, ciprofloxacina $(5 \mu \mathrm{g})$, trimetoprim/sulfametoxazol $(25 \mu \mathrm{g})$, gentamicina $(10 \mu \mathrm{g})$, norfloxacina $(10 \mu \mathrm{g})$ e ceftazidima $(30 \mu \mathrm{g})$.

Frente às bactérias Gram-positivas, foram testados os seguintes antimicrobianos: oxacilina $(1 \mu \mathrm{g})$, vancomicina $(30 \mu \mathrm{g})$, ampicilina $(10 \mu \mathrm{g})$, ciprofloxacina $(5 \mu \mathrm{g})$, trimetoprim/ sulfametoxazol $(25 \mu \mathrm{g})$ e linezolida $(30 \mu \mathrm{g})$.

Os testes de sensibilidade foram realizados pela automação MicroScan ${ }^{\circledR}$.

Análise dos dados. A resposta qualitativa para a Verificação da sensibilidade e resistência dos microrganismos aos antimicrobianos, em testes realizados pela automação $\left(\right.$ MicroScan $\left.{ }^{\circledR}\right)$ e difusão em disco (Kirby-Bauer) foi testada pelo $\chi^{2}$ : qui-quadrado ${ }^{8}$. 


\section{RESULTAD0S}

Foram analisadas 442 amostras de urina. Destas, houve 78 $(17,6 \%)$ resultados positivos para infecção do trato urinário, sendo que, em 67 (85,9\%) amostras 0 agente causador da ITU eram bactérias Gram-negativas e em 11 (14,1\%) Gram-positivas.

Os microrganismos isolados responsáveis pelas ITUs estão descritos na Tabela 1. A Escherichia coli foi o microrganismo prevalente $(67,9 \%)$ causador desta infecção.

Tabela 1 - Frequêencia de microrganismos causadores de ITU em mulheres atendidas no ambulatório do LAS/UCG, na cidade de Goiânia-G0, 2003.

\begin{tabular}{llrlll}
\hline & \multicolumn{2}{c}{ Freqüência } & & \multicolumn{2}{c}{ Freqüência } \\
\cline { 2 - 3 } \cline { 5 - 6 } Bactérias Gram-negativas & $n^{0}$ & $\%$ & Bactérias Gram positivas & $n^{0}$ & $\%$ \\
\hline Escherichia coli & 53 & 67,9 & Streptococcus agalactiae & 3 & 3,8 \\
Kebsiella oxytoca & 3 & 3,8 & Enterococcus sp & 2 & 2,5 \\
Citrobacter koseri & 2 & 2,5 & Staphylococcus aureus & 2 & 2,5 \\
Proteus mirabillis & 2 & 2,5 & Staphylococcus epidermidis & 2 & 2,5 \\
Cedecea davisae & 1 & 1,3 & Staphylococcus haemolyticus & 1 & 1,3 \\
Enterobacter aerogenes & 1 & 1,3 & Staphylococcus saprophyticus & 1 & 1,3 \\
Kebsiella pneumoniae & 1 & 1,3 & & & \\
Kebsiella sp & 1 & 1,3 & & & \\
Salmonella sp & 1 & 1,3 & & & \\
Serratia liquefaciens & 1 & 1,3 & & & \\
Serratia marcescens & 1 & 1,3 & &
\end{tabular}

Os microrganismos Gram-negativos se mostraram resistentes em maior índice a amoxicilina $(74,6 \%)$, seguido pelo trimetoprim/sulfametoxazol com $41,8 \%$, ciprofloxacina e norfloxacina com 13,4\% de resistência, ceftazidima com $6 \%$ e gentamicina com $1,5 \%$ de resistência.

As bactérias Gram-positivas mostraram-se resistentes em maior índice à ampicilina (72,7\%), seguido pela ciprofloxacina com $36,4 \%$, oxacilina com $27,3 \%$ e, trimetoprim/sulfametoxazol, vancomicina e linezolida com $18,2 \%$ de resistência dos microrganismos a estes antimicrobianos.

Tomando o método de difusão em disco (Kirby-Bauer) como resultado esperado, por ser aquele mais tradicionalmente utilizado, e confrontando com o método automatizado $\left(\right.$ MicroScan $\left.^{\circledR}\right)$, o resultado obtido pelo teste $\chi^{2}$ não revelou diferença significativa entre os métodos analisados ( $\mathrm{P}>0,05$; $\chi^{2}$ calculado $=0,228 ; \chi^{2}$ tabelado (a 5\%, GL11) $=19,68$ ).

No perfil de sensibilidade antimicrobiana das bactérias Grampositivas, 0 teste $\chi^{2}$ também não revelou diferença significativa entre as duas metodologias empregadas $\left(\mathrm{P}>0,05 ; \chi^{2}\right.$ calculado $=0,016 ; \chi^{2}$ tabelado $(\alpha 5 \%$, GL 11 $\left.)=19,68\right)$.

\section{DISCUSSÃ0}

A incidência de urinoculturas positivas encontrada neste estudo se assemelha aos trabalhos de Rieger e Horta ${ }^{35} \mathrm{no}$ Rio Grande do Sul, e Chaves e cols ${ }^{10}$ no Ceará. Outros autores apresentam porcentagens que variam de 28,7 a $75 \%$, dependendo das características da população estudada ${ }^{162834}$. Aincidência de infecção do trato urinário pode variar de acordo com a condição socioeconômica, com a presença de diabetes melitus, com as condições de higiene, após as relações sexuais, na presença de automedicação e de anormalidades anatômicas do trato urinário com maior frequiência nas mulheres ${ }^{24}$.

A incidência percentual de microrganismos causadores de ITU observados no presente estudo são concordantes com relatos descritos na literatura ${ }^{1} 92028$. Parece haver variações de acordo com a região ou país, conforme relatado por Moges cols ${ }^{25}$, que afirmam que 28,5\% das ITUna Etiópia são causadas por bactérias Gram-positivas.

No presente estudo, a Escherichia coli foi o principal microrganismo prevalente causador de ITU(67,9\%), o que está de acordo com a literatura ${ }^{32} 3435$.

0 estudo epidemiológico dos uropatógenos e 0 estabelecimento do perfil da sensibilidade aos antimicrobianos são aspectos de relevância, pois podem ser significativamente diferentes por estarem associados a pressões seletivas locais ${ }^{35}$.

As bactérias identificadas neste estudo se mostraram resistentes em maior índice a amoxicilina e ampicilina, 74,6\% e 72,7\% respectivamente. Mesmo apresentando uma alta concentração urinária, estes antimicrobianos não são recomendados para tratamento das ITUs por causa da resistência e alta recorrência, se comparados a outros agentes, sendo esta resistência justificada principalmente pela produção de $\beta$-lactamases e por alterações nas proteínas de ligação das penicilinas ( PBPs) das bactérias. Na atualidade, para uma boa resposta terapêutica no caso das ITUs, deve-se utilizar a amoxicilina associada ao ácido clavulânico ${ }^{14}$.

A elevada resistência microbiana ao trimetoprim/ sulfametoxazol é justificada pelo fato de ser um antimicrobiano mais antigo, já muito utilizado em infecções, possivelmente de uma maneira indiscriminada e aleatória com a automedicação ${ }^{23}$.

Diversos estudos demonstram alta sensibilidade de bactérias Gram-negativas a ciprofloxacina e norfloxacina ${ }^{3112335}$. 0 certo grau de resistência encontrado neste estudo pode ser justificado, também, pelo uso inadequado, que acarretaria alterações cromossômicas ${ }^{23}$. Para infecções graves, causadas por Enterobacteriaceae, é recomendado o uso da gentamicina, esta droga inibe a síntese de proteínas ao bloquear a formação do complexo de iniciação, se ligando na porção 30S do ribossoma bacterian $0^{541}$.

Com 0 advento da resistência às penicilinas, foi produzida a oxacilina, sendo portanto, uma penicilina resistente às penicilinases. É um antimicrobiano $\beta$-lactâmico, isoxazolil penicilina, utilizada para tratamento de infecções causadas por S. aureus ${ }^{541}$. Entretanto, neste estudo, as bactérias Gram-positivas foram resistentes à oxacilina em $27,3 \%$ dos casos, resultado concordante aos trabalhos de Jones e cols ${ }^{15}$ e Arias cols 2 .

No caso de resistência das bactérias Gram-positivas à oxacilina, a vancomicina é o glicopeptídeo de escolha para tratamento da ITU. Esta droga inibe a síntese da parede celular bacteriana ao interromper a reação de transglicosilaçã $0^{30}$. Entretanto, segundo Oliveira cols ${ }^{29}$, no Brasil, o isolamento de Enterococcus sp resistentes à vancomicina foi relatado em vários hospitais e a sua 
frequiência de isolamento continua aumentando gradativamente. De acordo com Luh cols ${ }^{21}$ e Weiss cols ${ }^{42}$, os Staphylococcus coagulase negativo apresentam resistência a vancomicina em $5 \%$ dos casos.

A linezolida é uma droga promissora no tratamento de infecções por microrganismos Gram-positivos multirresistentes. É uma oxazolidinona que inibe a síntese protéica ao bloquear a formação do complexo de iniciação, se ligando na porção 50S do ribossoma bacterian $0^{33}$. Jones cols ${ }^{15}$ afirmaram que a linezolida é considerada sensível para 100\% dos estafilococos, $99,4 \%$ dos estreptococos e em $96 \%$ dos enterococos. Entretanto, neste trabalho, as bactérias Gram-positivas mostraram maior $(18,2 \%)$ resistência.

A maior resistência das bactérias Gram-positivas testadas à vancomicina e à linezolida (18,2\%) observada no presente estudo deve-se ao fato da amostragem ter sido pequena. Apenas 11 $(14,1 \%)$ pacientes com ITU tinham como agente desta infecção microrganismos Gram-positivos, destes, apenas dois apresentaram multirresistência. Por isso, este dado não condiz com relatos da literatura em que foram utilizadas uma grande amostragem para testar as bactérias frente a esses antimicrobianos s $^{152} 2942$.

As duas amostras que apresentaram resistência frente à vancomicina e a linezolida foram: uma de Enterococcus sp, e uma de Staphylococcus saprophyticus. Com baseneste trabalho, verificase que os microrganismos multirresistentes não estão limitados ao âmbito hospitalar, pois estas amostras provêm de pacientes atendidas em ambulatório, ou seja, indivíduos portadores destes microrganismos que podem estar disseminado-os na comunidade.

De acordo com os trabalhos de Steward cols ${ }^{40}$, Koeth cols ${ }^{17}$ e Azevedo cols ${ }^{4}$, as semelhanças entre os resultados, na identificação e no antibiograma, de microrganismos, utilizandose métodos automatizados (MicroScan ${ }^{\circledR}$ ) e difusão em disco (Kirby-Bauer), são superiores a 95\% dos casos analisados.

Com este estudo, pode-se concluir que a Escherichia coli foi o microrganismo mais freqüente $(67,9 \%)$ causador de infecção do trato urinário em mulheres ambulatoriais na Cidade de Goiânia, G0. As bactérias Gram-negativas são sensíveis em maior índice a ceftazidima e gentamicina e as Gram-positivas ao trimetoprim/sulfametoxazol, à vancomicina e à linezolida. A identificação e 0 perfil de suscetibilidade antimicrobiana dos microrganismos causadores de ITU podem ser realizados tanto por técnica automatizada como pelo método de difusão em disco, por não apresentarem diferenças significativas nos resultados.

\section{AGRADECIMENTOS}

Os autores agradecem ao Laboratório de Análises da Saúde da Universidade Católica de Goiás e ao Laboratório Pfizer.

\section{REFERÊNCIAS BIBLIOGRÁFICAS}

1. Abu-Shaqra Q. Occurrence and antibiotic sensitivity of Enterobacteriaceae isolated from a group of Jordanian patients with community acquired urinary tract infections. Cytobios 101:15-21, 2000.
2. Arias CA, Reyes J, Zúñiga M, Cortés L, Cruz C, Rico CL, Panesso D and on behalf of the Colombian Antimicrobial Resistance Group (RESCOL). Multicentre surveillance of antimicrobial resistance in enterococci and staphylococci from Colombian hospitals, 2001-2002. Journal of Antimicrobial Chemotherapy 51: 59-68, 2003.

3. Astal Z, Manama A, Sharif FA. Antibiotic resistance of bacteria associated with community-acquired urinary tract infections in the southern area of the Gaza Strip. Journal Chemotherapy 14:259-264, 2002.

4. Azevedo PA, Cícero AGD, Gonçalves ALS, Rowe F, Teixeira LM. Evaluation of an automated system for the identification and antimicrobial susceptibility testing of enterococci. Diagnostic Microbiology and Infectious Disease 40:157-161, 2001.

5. Barros E, Bittencourt H, Caramori ML, Machado A. Antimicrobianos, consulta rápida. Artmed, Porto Alegre, 2001.

6. Bauer AW, Kirby EM. Antibiotic Susceptibility Testing by Standardized Single Disk Method. American Journal of Clinical Pathology 45:493-496, 1966.

7. Castro MS, Pilger D, Ferreira MBC, Kopittke L. Trends in antimicrobial utilization in a university hospital, 1990-1996. Revista de Saúde Pública 36:553-558,2002.

8. Centeno AJ. Distribuição e teste de $x^{2}$. In: Curso de Estatística Aplicada à Biologia. UFG, Goiânia, p.193-209, 1999.

9. Colodner R, Keness Y, Chazan B, Raz R. Antimicrobial susceptibility of community-acquired uropathogens in northern Israel. International Journal of Antimicrobial Agents 18:189-192, 2001.

10. Chaves JM, Moreira AA, Cunha FA, Carvalho MJP, Menezes EA. Perfil de resistência e sensibilidade dos antimicrobianos utilizados em infecções urinárias no Hospital de Referência São Lucas da cidade de Crateús- Ceará. Revista Brasileira de Análises Clínicas 35( supl) : 38B, 2003.

11. Gordon KA, Jones RN. Susceptibility patterns of orally administered antimicrobials among urinary tract infection pathogens from hospitalized patients in North America: comparison report to Europe and Latin America. Results from the SENTRY Antimicrobial Surveillance Program (2000). Diagnostic Microbiology and Infectious Disease 45:295-301, 2003.

12. Gram C. Uberdie Isolirte Farbung der Schizomycetem in Schmitt-und Trockenpaparaten. Fortschritte der Medizin 2:185-189, 1884.

13. Gupta K, Hooton TM, Roberts PL, Stamm WE. Patient-Initiated Treatment of Uncomplicated Recurrent Urinary Tract Infections in Young Women. Annals of Internal Medicine 135:9-16, 2001.

14. Jancel T, Dudas V. Management of uncomplicated urinary tract infections. Western Journal Medicine 176:51-55, 2002.

15. Jones RN, Ballow CH, Biedenbach and the ZAPS Study Group Medical Centers. Multi-laboratory assessment of the linezolid spectrum of activity using the Kirby-Bauer disk diffusion method: Report of the Ziyvox ${ }^{\circledR}$ Antimicrobial Potency Study (ZAPS) in the United States. Diagnostic Microbiology and Infectious Disease 40: 59-66, 2001.

16. Kahlmeter G. The ECO-SENS Project: a prospective, multinational, multicentre epidemiological survey of the prevalence and anti microbial susceptibility of urinary tract pathogens - interim report. Journal of Antimicrobial Chemotherapy 46 ( supl I) : 15-22, 2000.

17. Koeth LM, King A, Knight H, May J, Miller LA, Phillips I, Poupard JA. Comparison of cation-adjusted Mueller- Hinton broth with Iso-Sensitest broth for the NCCLS broth microdilution method. Journal of Antimicrobial Chemotherapy 46: 369-376, 2000.

18. Koneman EW, Allen SD, Janda WM, Schreckenberger PC, Winn WCJ. Diagnóstico Microbiológico. Medsi, Rio de Janeiro, 2001.

19. Kunin CM. Detection, Prevention and Management of Urinary Tract Infections: A manual for the Physician, Nurse and Allied Health Worker, $2^{\text {nd }}$ edition, Lea \& Febiger, Philadelphia, 1974.

20. Lazarevic G, Petreska D, Pavlovic S. Antibiotic sensitivity of bacteria isolated from the urine of children with urinary tract infections from 1986 to 1995. Srpski Arhiv Za Celokupno Lekarstvo126:423-429, 1998.

21. Luh KT, Hsueh PR, Teng LJ, Pan HJ, Chen YC, Lu JJ, Wu JJ, Ho SW. Quinupristin-Dalfopristin Resistance among Gram-Positive Bacteria in Taiwan Antimicrobic Agents and Chemotherapy 44: 3374-3380, 2000. 
22. Martino MDV, Toporovski J, Mímica MI. Bacteriological methods for screening urinary tract infection during childhood and adolescence. Jornal Brasileiro de Nefrologia 24:71-80, 2002.

23. Menezes EA, Araújo GN, Lopes HM, Costa EP, Nascimento IRO, Cunha FA. Perfil de Susceptibilidade a Norfloxacina e ao Sulfazotrim no tratamento de Infecções Urinárias causadas por Escherichia coli no Laboratório de Análises Clínicas de Esquadrão de Saúde da Base Aérea de Fortaleza. NewsLab ano IX 49:150-156, 2001.

24. Menezes EA, Melo OF, Oliveira IRN, Santos AS, Farias RB, Guerra ACP, Cunha FA. Bacteriúria assintomática em gestantes atendidas no Hospital da Base Aérea de Fortaleza. Laes \& Haes 140:84-96, 2003.

25. Moges AF, Genetu A, Mengistu G. Antibiotic sensitivities of common bacterial pathogens in urinary tract infections at Gondar Hospital, Ethiopia. Eastern African of Medicine Journal 79: 140-142, 2002.

26. Moreira MAA, Costa FS, Nogueira NAP. Bacteriúria assintomática em gestantes atendidas no Centro de Saúde Ambulatorial Abdornal Machado (CESA-AM) em Crateús, CE. Revista Brasileira de Análises Clínicas 35( supl): 41B, 2003.

27. National Committee for Clinical Laboratory Standards. Methods for dilution antimicrobial susceptibility tests for bacteria that grow aerobically. Documents M7-A6 and M100-S13. Wayne, Pa, USA, 2003.

28. Navaneeth BV, Belwadi S, Suganthi N. Urinary pathogens resistance to common antibiotics: a retrospective analysis. Tropical Doctor 32:20-22, 2002.

29. Oliveira GA, Atobe JH, Mamizuka EM. Enterococcus spp: Importância clínica, fatores de virulência, resistência aos antimicrobianos e diagnóstico laboratorial. Laes\&Haes 131:88-111, 2001

30. Oliveira GA, Levy CE, Mamizuka EM. Staphylococcus aureus with intermediate resistance to vancomycin: mechanisms of resistance, laboratory detection and perspectives of appearance in Brazil. Jornal Brasileiro de Patologia 36:96-102, 2000.

31. Ortiz V, Maia RS. Como Diagnosticar e Tratar Infecções do trato urinário. Revista Brasileira de Medicina 56:149-155, 1999.
32. Palma PCR, Dambros M. Cistites na mulher. Revista Brasileira de Medicina 59:346-350, 2002

33. Perry CM, Jarvis B. Linezolid: a review of its use in the management of serious Gram-positive infections. Drugs 61: 525-551, 2001

34. Ribeiro AS, Costa AM, Soto FS, Silva MQ, Silva RP. Estudo e prevalência de bactérias isoladas em urinoculturas de pacientes ambulatoriais. Revista Brasileira de Análises Clínicas 35( supl) :33B, 2003.

35. Rieger A, Horta JA. Prevalência de patógenos bacterianos e perfil de sensibilidade aos antimicrobianos em uroculturas de amostras comunitárias Revista Brasileira de Análises Clínicas 35( supl) : 37B, 2003.

36. Silva CHP. Elaboração e avaliação de um novo meio de cultura para a detecção e identificação presuntiva de enterobactérias produtoras de $\beta$ lactamase de espectro estendido (ESBL). Laes \& Haes 127: 148-160, 2000.

37. Sobel JD, Kaye D. Urinary tract infections. In: Mandel GL, Douglas RG, Bennett JE (eds) Principles and practice of infectious diseases. John Wiley\&Sons, New York, p.582, 1990.

38. Stamm AMNF. Fatores de risco e incidência da infecção do trato urinário relacionada a cateterização vesical. Estudo observacional de coorte contemporânea, não controlado. Dissertação de mestrado, Universidade Federal de Santa Catarina, Florianópolis, SC, 1994.

39. Stamm WE. Cystitis and Urethritis - In: Schrier RW, Gottschalk CW. Diseases of the kidney. $6^{\text {th }}$ edition, Little Brown and Company, Boston, USA, p.895912, 1996.

40. Steward CD, Stocker SA, Swenson JM, O'Hara CM, Edwards JR, Gaynes RP, McGowan Jr JE, Tenover FC. Comparison of Agar Dilution, Disk Diffusion, MicroScan, and Vitek Antimicrobial Susceptibility Testing Methods to Broth Microdilution for Detection of Fluoroquinolone-Resistant Isolates of the Family Enterobacteriaceae. Journal of Clinical Microbiology 37: 544-547, 1999.

41. Tavares W. Manual de antibióticos e quimioterápicos. Artmed, São Paulo, 2001.

42. Weiss K, Rouleau D, Laverdiere M. Cystitis due to vancomycin-intermediare S. saprophyticus. Journal of Antimicrobial Chemotherapy 37:1039-1040, 1996. 